\title{
Prevalence and Prognosis of Relapse of Nephroblastoma at the Pediatric Oncology Unit of Bamako
}

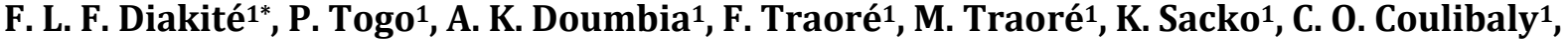 \\ N. L. Traoré1, A. Touré1, B. Maiga1 ${ }^{1}$, L. N. Sidibé1, D. Konaté1, A. Diall1, A. Dembélé1, \\ C. O. Coulibaly², C. B. Traoré ${ }^{3}$, B. Togo ${ }^{1}$
}

${ }^{1}$ Department of Pediatrics, CHU Gabriel Toure, Bamako, Mali

${ }^{2}$ Department of Pediatric Surgery, CHU Gabriel Toure, Bamako, Mali

${ }^{3}$ Department of Pathology Anatomy and Cytology, CHU Point G, Bamako, Mali

Email: *leoniediakite@gmail.com

How to cite this paper: Diakité, F.L.F., Togo, P., Doumbia, A.K., Traoré, F., Traoré, M., Sacko, K., Coulibaly, C.O., Traoré, N.L., Touré, A., Maiga, B., Sidibé, L.N., Konaté, D., Diall, A., Dembélé, A., Coulibaly, C.O., Traoré, C.B. and Togo, B. (2019) Prevalence and Prognosis of Relapse of Nephroblastoma at the Pediatric Oncology Unit of Bamako. Open Journal of Pediatrics, 9, 317-325.

https://doi.org/10.4236/ojped.2019.94031

Received: November 13, 2019

Accepted: December 7, 2019

Published: December 10, 2019

Copyright $\odot 2019$ by author(s) and Scientific Research Publishing Inc. This work is licensed under the Creative Commons Attribution International License (CC BY 4.0).

http://creativecommons.org/licenses/by/4.0/

\begin{abstract}
The nephroblastoma is the third pediatric cancer in Mali, this study aimed to describe the prevalence and prognosis of nephroblastoma relapses. Methods: It was a descriptive retrospective study over a 10 -year period from January 2005 to March 2015. We collected children aged 0 to 15 years followed for relapse of nephroblastoma in the pediatric oncology unit of university hospital center (UHC) Gabriel Toure. Results: The frequency of relapse of nephroblastoma was $7.4 \%$ ( 19 cases) whose mean age was 42 months with a sex ratio of 1.3. The relapse occurred before the end of the postoperative course in $16 \%$ of cases (3 patients). It was local recurrence in $52 \%$ of cases (10 patients), pulmonary $16 \%$ (3 patients), and hepatic $11 \%$ (2 patients). According to the SIOP classification, $47 \%$ of patients were diagnosed in stage III ( 9 patients) and $21 \%$ (4 patients) in stage IV. The tumor was high risk in $37 \%$. Palliative chemotherapy was performed in $63 \%$ of the patients (12 patients) and the remaining seven were put on a high risk diet. The overall survival at 5 years was $21 \%$ or 4 patients. Conclusion: Our results showed all the difficulties in the management of nephroblastoma relapses in our context.
\end{abstract}

\section{Keywords}

Nephroblastoma, Relapse, Mali

\section{Introduction}

Nephroblastoma or Wilms tumor is the most common pediatric renal tumor [1]. 
Advances in Wilms tumor management have been considered one of the historic success stories in oncology. Overall survival rates increased from $30 \%$ in the 1930 s to more than $90 \%$ in the current era, with improvements in surgical techniques, the introduction of chemotherapy and the discovery of radiosensitivity [2]. The incidence of relapse is between $10 \%-15 \%$ of patients with histologically favorable tumor after first-line treatment. This rate increases to $50 \%$ for patients with histologically unfavorable tumors [3] [4] [5]. Post-relapse survival of the disease remains inferior with a poor prognosis [4] [6] [7]. The relapsed Wilms tumor is clinically heterogeneous as is their sensitivity to chemotherapy. Results remain poor for the subgroup of patients with histologically unfavorable tumors, relapse at less than 12 months after diagnosis, and initial response to limited chemotherapy [3]. In developed countries survival is between $32 \%-60 \%$ [8] [9] [10] [11]. While in low-income countries, survival rates are much lower, and even lower in sub-Saharan Africa, ranging from $11 \%$ to 50\% [12] [13] [14] [15] [16], due to delayed diagnosis, malnutrition, poverty, discontinuation of treatment and very limited access to radiation therapy in these areas. Mali, with its socio-economic conditions, is very different from the developed world because of poverty, the shortage of hospital resources, the presentation of patients at a late stage, and the absence of data on cases of relapse of nephroblastoma after ten years of opening the pediatric oncology unit, hence the initiation of this study which aims to assess, the prevalence and prognosis relapses of nephroblastoma in Bamako.

\section{Material and Methods}

The oncology unit of the pediatric department of Gabriel Toure University Hospital in Bamako was the focus of our study. It is one of the pilot units of the French-African Group of Pediatric Oncology (FAGPO) in the management of pediatric cancers in the country and neighboring countries (Burkina Faso, Guinea Conakry ...). It is composed of 8 individual rooms of hospitalization, 3 administrative offices, a room of care, a room of preparation of chemotherapy with Hood and a hospital of day. The service activities are provided by four pediatric oncologists, four oncology nurses, a data manager and trainee physicians. We performed a descriptive study on the nephroblastoma cohort with retroprospective collection of data over a 10-year period (from January 1, 2005 to March 31, 2015). This study concerned all children aged 0 - 15 years followed for nephroblastoma in the Pediatric Oncology Unit of the Department of Pediatrics of UHC Gabriel Toure for relapse of nephroblastoma confirmed by abdominal ultrasound, chest X-ray or thoracic computed tomography. In total, we recorded 19 cases of relapse during the study period. All cases of nephroblastoma relapses that did not consult the unit and/or whose file was inoperable or lost were excluded. All patients had benefited from pre- and postoperative chemotherapy according to the protocol established by the French-African group of pediatric oncology (GFAOP) stemming from the 9th protocol SIOP (International Society of Pediatric Oncology) (2001) and adapted to the context of African countries 
after the study of feasibility in 8 pilot units across the African continent (Tunis, Algiers, Oran, Rabat, Casablanca, Yaoundé and Tananarive) [13]. Patients with localized nephroblastoma received 4 weeks of Vincristine and Actinomycin D cures prior to surgery, whereas patients with metastases received a 6-week preoperative course with Vincristine, Actinomycin D and Doxorubicin. In all cases, the continuation of treatment was a function of the local stage defined postoperatively:

- Stage I: adjuvant chemotherapy with 2 drugs (Vincristine, Actinomycin D).

- Stage II: adjuvant chemotherapy with 3 drugs (Vincristine, Actinomycin D, Adriamycin).

- Stage III: radiotherapy and adjuvant chemotherapy with 3 drugs.

- Stage IV: pre- and post-operative 3-drug chemotherapy with excision or irradiation of metastases in case of incomplete regression.

- Stage V: bilateral partial nephrectomy and treatment according to the local stage on each side.

- Treatment for relapse included alternating cyclophosphamide/Etoposide and Carboplatin/Etoposide treatments, surgery, and radiotherapy.

- None of our patients had received radiation therapy.

We defined relapse as the reappearance of the first cancer after a period of complete radiological or ultrasound remission. Depending on its location, we will talk about relapse: local (at the same place as the starting cancer); regional (lymph node metastases); at a distance, in another organ (liver, lungs, bone, brain). The method used to detect relapses was planned surveillance imaging (abdominal ultrasound, chest X-ray, abdominal and thoracic computed tomography). All patients had a blood count, liver and kidney function tests. Complete remission was defined as a lack of evidence of the disease clinically and on imaging. The survival rate was defined as the time interval between the date of diagnosis and the date of death, from any cause or date of the last visit to the clinic. The variables studied were socio-demographic profile, clinical and paraclinical data, therapeutic and evolutionary aspects. Analysis and data processing were done with SPSS software version 20.0

\section{Ethical considerations}

The patients treated according to the protocol of the French-African Group of Pediatric Oncology (FAGPO) validated by the Ethics Committee of the Faculty of Medicine and Odonto-Stomatology of Bamako. As with any retrospective study, we have requested access to medical records from the Pediatric Oncology Unit Head.

\section{Results}

\section{Prevalence}

During the study period, 1578 cases of pediatric cancers were diagnosed in the pediatric oncology unit. Among these patients there were 256 cases of nephroblastoma, a frequency of $16.2 \%$, and nineteen children relapsed or $7.4 \%$. Nephroblastoma ranked third after lymphoma and retinoblastoma, which accounted 
for $33.5 \%$ and $24.7 \%$ respectively.

\section{Sociodemographic characteristics}

Nearly half of the fathers $(47.4 \%)$ did not receive any type of education and they were peasants or workers in $73.7 \%$ of the cases. The majority of mothers, $89.4 \%$, were housewives and $73.7 \%$ had no education. Among them, $42 \%$ lived outside the city of Bamako and $58 \%$ had a low socioeconomic level.

\section{Clinical characteristics}

The majority of patients (95\%) were older than 2 years old. The average age was 42 months. The sex ratio was 1.3 . In more than two thirds of cases $(74 \%)$, the initial tumor was diagnosed more than 90 days after the onset of symptoms. The primary reason for consultation was a large abdominal mass (47\%). Clinically, the children had a general condition that was little altered (74\%) and malnutrition was present in $16 \%$ of the patients. Metastases were observed at diagnosis in $21 \%$ of cases. They were pulmonary in $16 \%$ of cases, anemia in thirteen patients $(68.4 \%)$ and arterial hypertension in three patients (16\%). The abdominal ultrasound showed a retro-peritoneal mass in all children that was associated with secondary hepatic localization in two patients $(11 \%)$ and the chest $\mathrm{X}$-ray showed pulmonary localization in three children. All patients had preoperative chemotherapy and the interval between cures was missed in four patients $(21 \%)$. At the time of surgery, all patients had had a nephrectomy; nine patients $(47.3 \%)$ were in stage III surgical and four patients $(21 \%)$ in stage IV. Seven patients (37\%) had a high-risk histologic tumor, seven patients $(31 \%)$ had an intermediate risk and one room had not been analyzed because it had been buried by the parents.

\section{Prognosis of relapses}

The average time to relapse was 10 months after nephrectomy; $16 \%$ of patients had recurred before the end of postoperative chemotherapy and $84 \%$ of relapses after this treatment. The relapse was local (53\%), pulmonary (32\%), hepatic (21\%) and cerebral (5\%). The treatment consisted of high-risk chemotherapy with four molecules (cyclophosphamide, Doxorubicin, Carboplatin, Etoposide) in $37 \%$ of cases and palliative treatment in $63 \%$ of cases. No patient had received radiation therapy. The evolution was marked by death in $58 \%$ of cases; $21 \%$ were lost to follow-up and $21 \%$ had survived the relapse of which $5 \%$ were in continuous remission. The toxicities of chemotherapy were mainly hematological (8/19), digestive (7/19) and cutaneous (1/19). Deaths occurred very early in a context of severe sepsis (10/19).

\section{Study Population by Fate}

Among these patients, there is not a big difference between the two sexes in the group of deceased (Male 6/11 death and female 5/11 death) as well as the age of the patients. For the delay of relapses on the 11 deceased patients 7 patients had a duration less than 3 months. More than half (6/11 of the deceased) are seen in advanced stage III disease. The no blastematous histological type had a poor prognosis (8/11 of the deceased). Thus the advanced stage of the disease and the histological type are of poor prognosis (Table 1 ). 
Table 1. Characteristics of the study population.

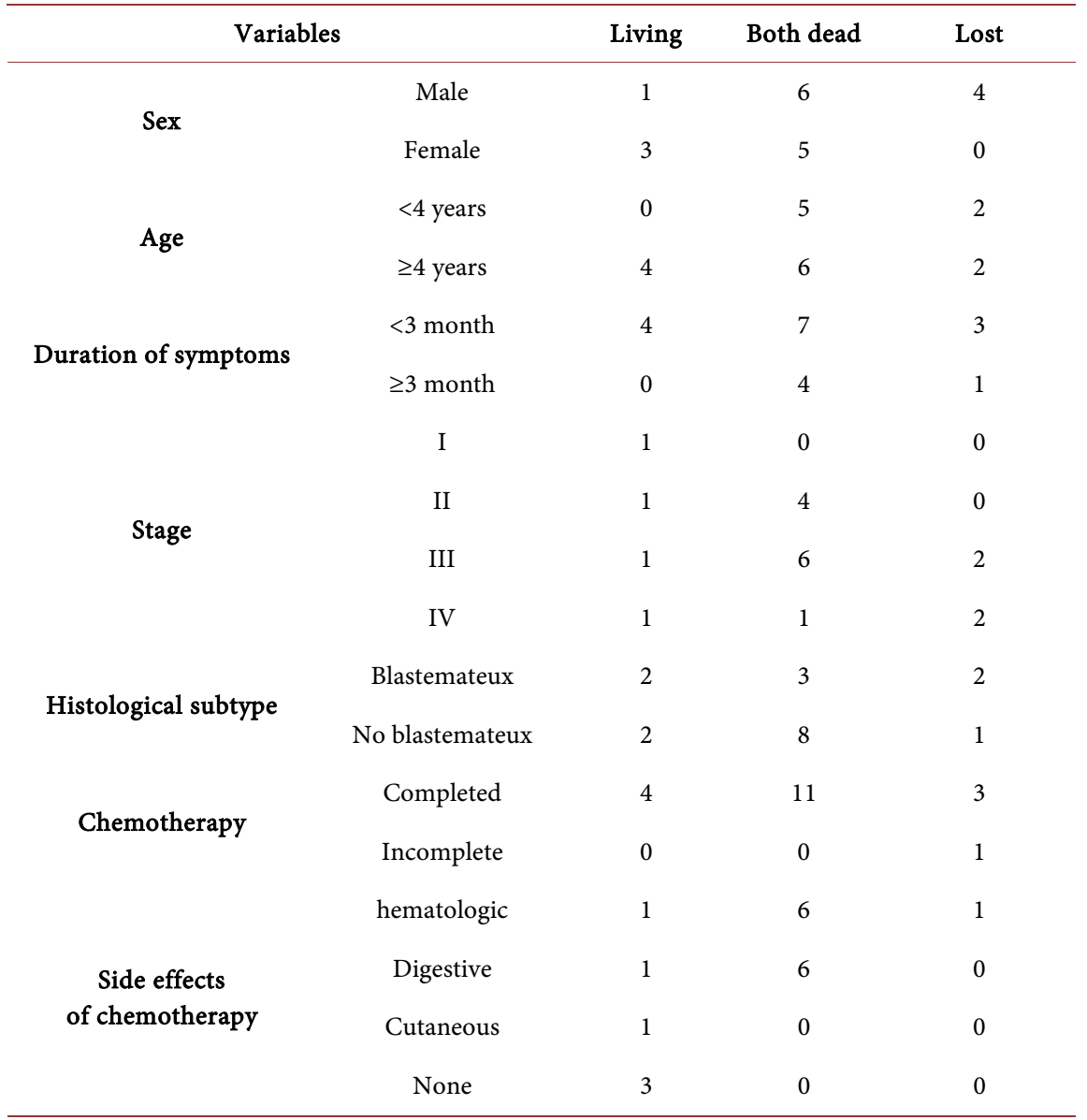

\section{Comments and Discussion}

\section{Limitations and difficulties encountered}

This is a retrospective study that presents a number of difficulties.

- The insufficiency and the no exploitability of certain files.

- The majority of patients who have not done all the necessary additional examinations.

- The insufficiency of the technical platform for the realization of certain complementary and therapeutic examinations.

We did not record a loss of file.

\section{Relapse rate}

The relapse rate of nephroblastoma was estimated at $7.4 \%$. This value could be underestimated because of the rather high rate of loss of sight due mainly to poverty and the distance from the household treatment site [17]; our unit is the only one to detect, treat and monitor pediatric cancers in Mali. This rate is lower than that found by the 4th National Wilm's Tumor Study Group (NWTS) and the 9th SIOP (International Society of Pediatric Oncology), which give $11 \%$ and $10 \%$, respectively [18]. In Egyptian studies, relapse rates are between $17 \%$ and $24 \%$ [18] [19]. 


\section{Clinical and pathological features of relapses}

In our series, cases of relapse of the Wilms tumor were more common in boys than in girls. In Europe, the gender ratio is close to 1, while in the United States there is a female predominance [7] [20]. Male sex is one of the risk factors for recurrence [7]. Cooperative group studies have shown that increasing the patient's age is associated with an increased risk of non-metastatic Wilms tumor recurrence [21] [22]. We found that the vast majority of $95 \%$ of our patients were over 2 years old. The average age of the patients was 42 months, which is very close to the age of onset of the first disease. The majority of our patients (68\%) were in advanced stages (III or IV) and thirteen patients (68\%) had intermediate or anaplastic tumors. According to the literature, about $10 \%$ to $15 \%$ of favorable histology's and 50\% of anaplastic histology's recur [23] [24] [25] [26]. The poor prognostic factors found in the literature are the advanced clinical stage of the disease, the anaplastic histological type and the initial chemoresistance, these factors increase the risk of progression or recurrence of the disease [3] [4] [18] [27].

\section{Prognosis of relapses}

Early relapses occurred in our setting, usually occurring in less than 12 months after nephrectomy. In a 1995-2015 SIOP study by Brok, J. et al., 80\% of relapses occur within 24 months of nephrectomy, 13\% within 24 to 60 months and a very late relapse (>60 months) is recorded at 7\% [7]. In our series, relapses were most often local (52\%), contrary to data from the literature where they most often occur in the lungs ( $60 \%$ to $70 \%$ ), in the abdomen (10\% to $30 \%)$ and more rarely in the bones or the brain [7] [19] [23]. The risk of local or metastatic relapse increases with incomplete excision [28]. According to several studies, the predictive factors for shorter survival are a relapse in less than six months between nephrectomy and relapse, stages II, III and IV and high-risk histology, in addition to the male sex, advanced age, multiple sites of relapse and increased tumor volume [7] [21]. These classic factors could also be associated with delay in diagnosis and management, insufficient means of detection and timidity of multidisciplinary consultation meetings (MDC). According to Lemerle et al., the problem in the management is not the aggressiveness of the treatments, but their thoroughness and a necessary collaboration between radiologists, chemotherapists, surgeons, pathologists and radiotherapists [29]. In our study, the mortality rate was high in case of relapse (58\%). Post-relapse survival rates of $50 \%$ to $60 \%$ are reported with Ifosphamide, Etoposide and Carboplatin (ICE) chemotherapy [30]. Given the narrow outcome of the GFAOP strategy, the individualization of the treatment depending on the size and extent of the tumor, the general condition of the patient and the surgeon's experience would probably be needed to achieve better results in the future our context [31]. The role of surgery and radiotherapy for recurrence of nephroblastoma need to be precisely defined, although some recent studies suggest that complete surgical resection and radiotherapy of previously untreated fields may improve survival [30]. 


\section{Conclusion}

The study showed the difficulty of managing nephroblastoma relapses in the Malian context. The relapse rate was underestimated. These recurrences of nephroblastoma were generally early, local and of adverse histology. The high-grade Wilms tumor requires further study, and continuous monitoring of patients in remission will identify and quantify late complications. These results could be improved by multidisciplinary management. We recommend monitoring within 24 months of nephrectomy by regular follow-up every 3 months and have easier access to radiation therapy.

\section{Conflicts of Interest}

The authors declare no conflicts of interest regarding the publication of this paper.

\section{References}

[1] Grovas, A., Fremgen, A., et al. (1997) The National Cancer Data Base Report on Patterns of Childhood Cancers in the United States. Cancer, 80, 2321-2332. https://doi.org/10.1002/(SICI)1097-0142(19971215)80:12<2321::AID-CNCR14>3.0. CO;2-W

[2] Metzger, M.L. and Dome, J.S. (2005) Current Therapy for Wilms' Tumor. The Oncologist, 10, 815-826. https://doi.org/10.1634/theoncologist.10-10-815

[3] Grundy, P., Breslow, N., Green, D.M., et al. (1989) Prognostic Factors for Children with Recurrent Wilms' Tumor: Results from the Second and Third National Wilms' Tumor Study. Journal of Clinical Oncology, 7, 638-647. https://doi.org/10.1200/JCO.1989.7.5.638

[4] Campbell, A.D., et al. (2004) Treatment of Relapsed Wilms' Tumor with High-Dose Therapy and Autologous Hematopoietic Stem-Cell Rescue: The Experience at Children's Memorial Hospital. Clinical Oncology, 22, 2885-2890. https://doi.org/10.1200/JCO.2004.09.073

[5] Malogolowkin, M., Spreafico, F., Dome, J.S., et al. (2013) Incidence and outcomes of patients with late relapse of Wilms' tumour. Pediatric Blood \& Cancer, 60, 1612-1615. https://doi.org/10.1002/pbc.24604

[6] Graf, N., Tournade, M.F. and de Kraker, J. (2000) The Role of Preoperative Chemotherapy in the Management of Wilms' Tumor. The SIOP Studies. Urologic Clinics of North America, 27, 443-454. https://doi.org/10.1016/S0094-0143(05)70092-6

[7] Brok, J., Lopez-Yurda, M., Tinteren, H.V., et al. (2018) Relapse of Wilms' Tumour and Detection Methods: A Retrospective Analysis of the 2001 Renal Tumour Study Group-International Society of Paediatric Oncology Wilms' Tumour Protocol Database. The Lancet Oncology, 19, 1072-1081.

https://doi.org/10.1016/S1470-2045(18)30293-6

[8] Garaventa, A., Hartmann, O., Benard, J.L., et al. (1994) Autologous Bone Marrow Transplantation for Pediatric Wilms' Tumor: The Experience of the European Bone Marrow Transplantation Solid Tumor Registry. Medical and Pediatric Oncology, 22, 11-14. https://doi.org/10.1002/mpo.2950220103

[9] Pein, F., Michon, J., Valteau-Couanet, D., et al. (1998) High-Dose Melphalan, Etoposide, and Carboplatin Followed by Autologous Stem-Cell Rescue in Pediatric 
High-Risk Recurrent Wilms' Tumor: A French Society of Pediatric Oncology Study. Journal of Clinical Oncology, 16, 3295-3301. https://doi.org/10.1200/JCO.1998.16.10.3295

[10] Dagher, R., Kreissman, S., Robertson, K., et al. (1998) High Dose Chemotherapy with Autologous Peripheral Blood Progenitor Cell Transplantation in an Anephric Child with Multiply Recurrent Wilms' Tumor. Journal of Pediatric Hematology/Oncology, 20, 357-360. https://doi.org/10.1097/00043426-199807000-00016

[11] Kremens, B., Gruhn, B., Klingebiel, T., et al. (2002) High-Dose Chemotherapy with Autologous Stem Rescue in Children with Nephroblastoma. Bone Marrow Transplantation, 30, 893-898. https://doi.org/10.1038/sj.bmt.1703771

[12] Israels, T., Borgstein, E., Pidini, D., et al. (2012) Management of Children with a Wilms Tumour in Malawi, Sub-Saharan Africa. Pediatric Blood \& Cancer, 59, 636-641. https://doi.org/10.1002/pbc.24088

[13] Moreira, C., Nachef, M.N., Ziamati, S., et al. (2012) Treatment of Nephroblastoma in Africa: Results of the First French African Pediatric Oncology Group (GFAOP) Study. Pediatric Blood \& Cancer, 58, 37-42. https://doi.org/10.1002/pbc.23284

[14] Abuidris, D.O., Elimam, M.E., Nugud, F.M., et al. (2008) Wilms Tumour in Sudan. Pediatric Blood \& Cancer, 50, 1135-1137. https://doi.org/10.1002/pbc.21547

[15] Israels, T. (2012) Wilms Tumor in Africa: Challenges to Cure. Pediatric Blood \& Cancer, 58, 3-4. https://doi.org/10.1002/pbc.23334

[16] Wilde, J.C., Lameris, W., van Hasselt, E.H., et al. (2010) Challenges and Outcome of Wilms' Tumour Management in a Resource-Constrained Setting. African Journal of Paediatric Surgery, 7, 159-162. https://doi.org/10.4103/0189-6725.70416

[17] Togo, B. (2014) Épidémiologie et pronostic des cancers pédiatriques au CHU Gabriel-Touré de Bamako (Mali). Médecine et Santé Tropicales, 24, 68-72. https://doi.org/10.1684/mst.2014.0291

[18] Sherif, F., et al. (2008) Multidisciplinary Approach to Wilms' Tumor: A Retrospective Analytical Study of 53 Patients. Journal of the Egyptian National Cancer Institute, 20, 410-423.

[19] Abd El-Aal, H.H., et al. (2005) Wilms' Tumor: The Experience of the Pediatric Unit of Kasr El-Aini Center of Radiation Oncology and Nuclear Medicine (NEMROCK). Journal of the Egyptian National Cancer Institute, 17, 308-314.

[20] Yildiz, I., Yuksel, L., Ozkan, A., et al. (2000) Multidisciplinary Approach to Wilm's Tumor: 18 Years of Experience. Japanese Journal of Clinical Oncology, 30, 17-20. https://doi.org/10.1093/jjco/hyd001

[21] Breslow, N., Sharples, K., Beckwith, J.B., Takashima, J., et al. (1991) Prognostic Factors in Nonmetastatic, Favorable Histology Wilms' Tumor. Results of the Third National Wilms' Tumor Study. Cancer, 68, 2345-2353. https://doi.org/10.1002/1097-0142(19911201)68:11<2345::AID-CNCR2820681103> 3.0.CO;2-T

[22] Pritchard-Jones, K., Kelsey, A., Vujanic, G., et al. (2003) Older Age Is an Adverse Prognostic Factor in Stage I, Favorable Histology Wilms' Tumor Treated with Vincristine Monochemotherapy: A Study by the United Kingdom Children's Cancer Study Group, Wilms' Tumor Working Group. Journal of Clinical Oncology, 21, 3269-3275. https://doi.org/10.1200/JCO.2003.01.062

[23] Pein, F., Sarnacki, S., Boccon-Gibod, L., et al. (2008) Néphroblastome. In: Kalifa, C., Oberlin, O., Pein, F., Hartmann, O. and Lemerle, J., Eds., Cancers de Penfant, Flammarion, Paris, 187-202. 
[24] Spreafico, F., Pritchard, J.K., Malogolowkin, M.H., et al. (2009) Treatment of Relapsed Wilms Tumors: Lessons Learned. Expert Review of Anticancer Therapy, 9, 1807-1815. https://doi.org/10.1586/era.09.159

[25] Irtan, S., Sznadjer, B., Von Tinteren, H., et al. (2015) Is Biopsy a Risk Factor for Wilms Tumor Local Recurrence in the SIOP WT 2001 Trial ? Pediatric Blood \& Cancer, 62, 162.

[26] Irtan, S., Jitlal, M., Bate, J., et al.. (2015) Risk Factors for Local Recurrence in Wilms Tumour and the Potential Influence of Biopsy-the United Kingdom Experience. European Journal of Cancer, 51, 225-232. https://doi.org/10.1016/j.ejca.2014.10.026

[27] Green, D.M. (2004) The Treatment of Stages I-IV Favorable Histology Wilms Tumor. Journal of Clinical Oncology, 22, 1366-1372.

https://doi.org/10.1200/JCO.2004.08.008

[28] Mhamed, H. (2012) Cancer in Children: Practical Aspects. Morocco Printing, 86-95.

[29] Lemerle, J., Barsaoui, S., Harif, M., Hireche, K., et al. (2007) Le traitement des cancers de l'enfant en Afrique travaux du Groupe Franco-Africain d'Oncologie Pédiatrique. Medecine tropicale, 67, 497-504.

[30] Dome, J.S., Liu, T., Krasin, M., Lott, L., Shearer, P., Daw, N.C., et al. (2002) Improved Survival for Patients with Recurrent Wilms Tumor: The Experience at St. Jude Children's Research Hospital. Journal of Pediatric Hematologyl Oncology, 24, 192-198. https://doi.org/10.1097/00043426-200203000-00007

[31] Tongaonkar, H.B., Qureshi, S.S., et al. (2007) Wilms' Tumor: An Update. Indian Journal of Urology, 23, 458-466. https://doi.org/10.4103/0970-1591.36722 